Research Paper

\title{
Highly Frequent and Enhanced Injection Site Reaction Induced by Peripheral Venous Injection of Fosaprepitant in Anthracycline-Treated Patients
}

Yumiko Sato ${ }^{1}$, Masahiro Kondo ${ }^{2}$, Atsushi Inagaki ${ }^{3 凶}$, Hirokazu Komatsu ${ }^{4}$, Chika Okada ${ }^{5}$, Kumi Naruse ${ }^{6}$, Tomoyo Sahashi ${ }^{6}$, Junko Kuroda ${ }^{2}$, Hiroka Ogura ${ }^{3}$, Shiori Uegaki ${ }^{4}$, Tatsuya Yoshida 4 , Yoshinori Mori 4 , Hiroo Sawada 7, Shoichi Watanabe ${ }^{8}$, Hiroshi Sugiura 9 , Yumi Endo 10, Nobuyasu Yoshimoto 10, Tatsuya Toyama ${ }^{10}$, Shinsuke Iida ${ }^{11}$, Koichi Yamada ${ }^{1}$, Kazunori Kimura ${ }^{2}$ and Atsushi Wakita ${ }^{3}$

1. Department of Pharmacy, Nagoya City West Medical Center.

2. Department of Pharmacy, Nagoya City University Hospital.

3. Department of Hematology and Oncology, Nagoya City West Medical Center.

4. Division of Oncology, Nagoya City University Hospital.

5. Nursing department, Nagoya City West Medical Center.

6. Nursing department, Nagoya City University Hospital.

7. Department of Dermatology, Nagoya City West Medical Center.

8. Department of Geriatric and Environmental Dermatology, Nagoya City University Graduate School of Medical Sciences.

9. Department of Breast and Endocrine Surgery, Nagoya City West Medical Center.

10. Department of Oncology, Immunology and Surgery, Nagoya City University Graduate School of Medical Sciences.

11. Department of Medical Oncology and Immunology, Nagoya City University Graduate School of Medical Sciences.

$\square$ Corresponding author: Mr. Atsushi Inagaki, Department of Hematology and Oncology, Nagoya City West Medical Center, 1-1-1, Hirate-chou, Kita-ku, Nagoya, Aichi, 462-8508, Japan. Phone: +81-52-991-8121; Fax: +81-52-916-2038; E-mail: a.inagaki.21@west-med.jp

() Ivyspring International Publisher. This is an open-access article distributed under the terms of the Creative Commons License (http://creativecommons.org/ licenses/by-nc-nd/3.0/). Reproduction is permitted for personal, noncommercial use, provided that the article is in whole, unmodified, and properly cited.

Received: 2013.09.19; Accepted: 2014.02.26; Published: 2014.04.24

\begin{abstract}
Background: Fosaprepitant-associated injection site reaction (ISR) has been reported in patients treated with cisplatin, an irritant drug. We conducted this retrospective study to clarify the incidence and symptoms of fosaprepitant-associated ISR in patients treated with anthracycline.

Patients and methods: Fifty six patients receiving 159 injections administering doxorubicin/cyclophosphamide (AC), fluorouracil/epirubicin/cyclophosphamide (FEC), or rituximab/cyclophosphamide/doxorubicin/vincristine/prednisolone (R-) CHOP regimen through a peripheral vein at ambulatory treatment centers reviewed for this study from patients' medical records. Incidence of ISR was compared between 24 patients with fosaprepitant injection (fosaprepitant group) and 32 patients without fosaprepitant (control group). Frequency and symptoms of ISR per injection were also compared between $6 \mathrm{I}$ injections with fosaprepitant and 98 injections without fosaprepitant.

Results: Both the ISR incidence rate per patient and per injection were significantly higher in the fosaprepitant group than in the control group (67\% vs. $16 \% ; P=0.0002$, $34 \%$ vs. $8.2 \% ; P<0.000$ I, respectively). By multivariate analysis, fosaprepitant injection was found to be a significant independent variable correlated with ISR risk. Symptoms observed in $6 \mathrm{I}$ injections of fosaprepitant were pain $(n=14,23 \%)$, erythema $(n=10,16 \%)$, swelling $(n=6,10 \%)$, and delayed drip infusion $(n=6$, $10 \%$ ). After the observation period, no ISR occurred when the administration route was changed to central venous injection or oral aprepitant was administered despite the continuation of chemotherapy.

Conclusion: ISR occurred more frequently and severely when fosaprepitant was injected through the peripheral vein in patients treated with anthracyclines compared to those without fosaprepitant.
\end{abstract}

Key words: Antiemesis, anthracycline, fosaprepitant, injection site reaction, venous toxicity 


\section{INTRODUCTION}

Aprepitant, a neurokinin receptor antagonist (NK1RA), can improve the prevention of chemotherapy-induced nausea and vomiting (CINV) when administered with a 5-hydroxytryptamine-3 (5- $\left.\mathrm{HT}_{3}\right)$ receptor antagonist and dexamethasone in patients who are administered cisplatin or doxorubicin and cyclophosphamide (AC) for breast cancer. ${ }^{1-3}$ Fosaprepitant is a water-soluble, phosphorylated analog of aprepitant that is rapidly converted to aprepitant after intravenous administration. ${ }^{4}$ Bioequivalence of fosaprepitant (150 mg intravenously [i.v.]) with aprepitant (day 1, $125 \mathrm{mg}$; days 2 and 3, $80 \mathrm{mg}$ ) has recently been demonstrated. ${ }^{5}$ Due to this, fosaprepitant has been included into the clinical guidelines for treatment of CINV induced by highly emetogenic chemotherapy as proposed by the American Society of Clinical Oncology (ASCO), ${ }^{6}$ and the guidelines for treatment of CINV induced by moderate regimens, such as anthracycline and cyclophosphamide, and highly emetogenic chemotherapies as proposed by the Multinational Association of Supportive Care in Cancer (MASCC). ${ }^{7}$ Fosaprepitant was approved in the USA and Europe in January 2008 and in Japan in December 2011, and has been widely used in clinical practice.

Adverse events of fosaprepitant include asthenia, diarrhea, vomiting, anorexia, nausea, and hiccups. ${ }^{5}$ A previous study of patients treated with $\geq 70$ $\mathrm{mg} / \mathrm{m}^{2}$ cisplatin showed that the proportion of injection-related thrombophlebitis was significantly higher in the fosaprepitant group than in the aprepitant group. The proportion of side effects was not statistically different between the fosaprepitant and aprepitant groups except for injection-related thrombophlebitis. ${ }^{5}$ In the fosaprepitant group, the percentage of injection site reactions (ISR) was $2.2 \%$. Thirty events were measured in 25 patients, including two events of severe injection site pain, 9 events of moderate intensity, and 19 events of mild intensity. ${ }^{5}$

Anthracyclines, key drugs for the treatment of breast cancer and hematological malignancies, are vesicant drugs that cause severe tissue damage and necrosis by extravasation in tissues surrounding the injection site. Although a number of studies have described the proportion of ISR induced by fosaprepitant in patients treated with irritant drugs such as cisplatin, little is known about this effect in patients treated with vesicant drugs such as anthracyclines. Therefore, we conducted a retrospective study to determine the incidence and clinical state of ISR induced by fosaprepitant in patients treated with anthracycline.

\section{PATIENTS AND METHODS}

\section{Patients and Controls}

This study included 56 patients who were administered an anthracycline-containing regimen through a peripheral vein from November 2011 to April 2012 at two independent ambulatory treatment centers (Nagoya City West Medical Center and Nagoya City University Hospital, Japan). Twenty four patients who were administered fosaprepitant at least once during the observation period were categorized as the fosaprepitant group. The remaining 32 patients who underwent an anthracycline-containing regimen without fosaprepitant were categorized as the control group.
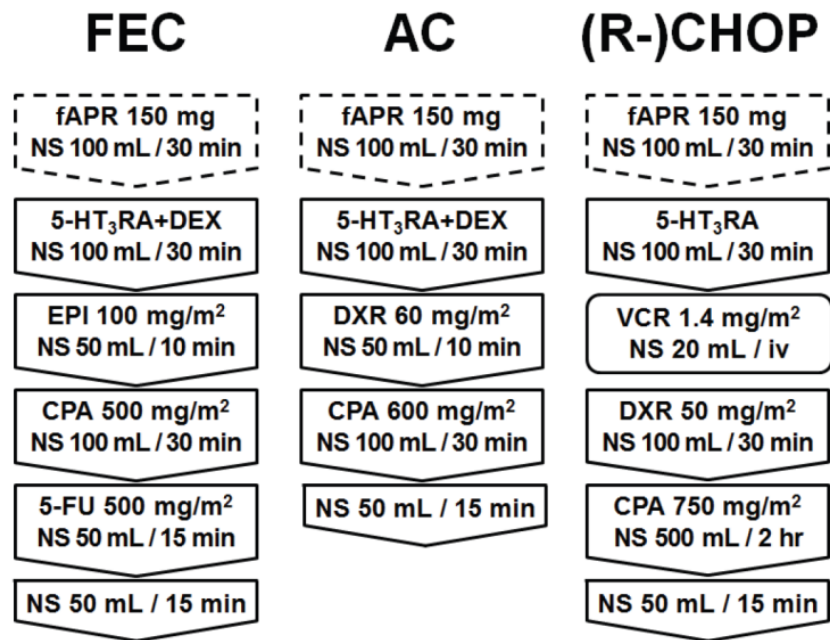

Figure I. Schema of treatment regimen. Fosaprepitant, $5-\mathrm{HT}_{3} \mathrm{RA}$ (FEC, AC: palonosetron $0.75 \mathrm{mg} /$ body, (R-)CHOP: granisetron $3 \mathrm{mg} /$ body), and/or dexamethasone $9.9 \mathrm{mg} /$ body were administered to patients (fosaprepitant group), while only 5- $\mathrm{HT}_{3} \mathrm{RA}$ (FEC, AC: palonosetron $0.75 \mathrm{mg} /$ body, (R-)CHOP: granisetron $3 \mathrm{mg} /$ body) and/or dexamethasone $9.9 \mathrm{mg} /$ body were administered to the control group via peripheral venous route for premedication of a particular chemotherapy (AC, FEC or (R-)CHOP). fAPR, fosaprepitant; NS, normal saline; 5- $\mathrm{HT}_{3} \mathrm{RA}$, 5-hydroxytryptamine-3 receptor antagonist; DEX, dexamethasone; EPI, epirubicin; DXR, doxorubicin; VCR, vincristine; iv, intravenous injection; CPA, cyclophosphamide; and 5-FU, 5-fluorouracil.

\section{Treatment protocols}

All patients underwent one of the following three regimens: doxorubicin/cyclophosphamide (AC), fluorouracil/epirubicin/cyclophosphamide (FEC), or rituximab/cyclophosphamide/doxorubicin/vincristine/prednisolone $\quad[(\mathrm{R}-) \mathrm{CHOP}] . \quad$ The schedules of FEC, AC and (R-)CHOP regimens are shown in Figure 1. In the fosaprepitant group, fosaprepitant was dissolved in $100 \mathrm{~mL}$ of normal saline $(1.5 \mathrm{mg} / \mathrm{mL})$, and subsequently, the solution was administered by drip into a peripheral vein for 30 minutes. Following fosaprepitant injection, $5-\mathrm{HT}_{3} \mathrm{RA}$ (FEC, AC: palonosetron $0.75 \mathrm{mg} /$ body, (R-)CHOP: granisetron $3 \mathrm{mg} /$ body) and/or dexamethasone 9.9 
$\mathrm{mg} /$ body was administered via the same peripheral venous route for premedication of a particular chemotherapy. In contrast, in the control group, fosaprepitant was not administered and only $5-\mathrm{HT}_{3} \mathrm{RA}$ (FEC, AC: palonosetron $0.75 \mathrm{mg} /$ body, (R-)CHOP: granisetron $3 \mathrm{mg} /$ body) and/or dexamethasone 9.9 $\mathrm{mg} /$ body were administered for premedication before each chemotherapy treatment. All chemotherapy drugs including anthracycline were administered via the peripheral vein in addition to antiemetic drugs.

\section{Definition and Evaluation of ISR}

In the present study, ISRs were evaluated according to the National Cancer Institute Common Toxicity Criteria (NCI-CTC), version 4.0 from the time of vascular puncture to the completion of all drug administration's. Injection site pain, erythema, swelling, or delayed drip infusion during administration of antiemetic and anticancer drugs were recorded as clinical symptoms of ISR. Treatment-required ISR was defined as ISR that required re-puncture, dermatological treatment (consultation with a dermatologist, the application of steroid ointment, or local injection to the ISR site), and/or discontinuation of administration. ISR was systematically evaluated for each injection as a daily practice by cancer chemotherapy nurses who had sufficient training to provide safe administration of anti-cancer drugs to cancer patients and who had obtained oncology nursing certification by the Japanese Nursing Association.

\section{Clinical data acquisition}

We used gender, age, body mass index (BMI), number of prior chemotherapy treatments, chemotherapy regimen, and injection site at administration as clinical variables to compare the background clinical variables and clinical status of ISR between the fosaprepitant and control groups, and to clarify the predictive factor of ISR. All clinical variables were collected from patients' medical records held by the ambulatory treatment centers. The investigation period of this study was between November 2011 and April 2012. This study was approved by the ethical review board at each participating facility.

\section{Statistical Analyses}

The clinical variables and incidence of ISR between the fosaprepitant and control groups were compared. The significance of differences in incidence of ISR and background clinical variables such as gender and number of prior chemotherapy treatments were compared using Fisher's exact test. Clinical variables such as distribution of chemotherapy regimen and injection site were compared by chi-square test. Age, BMI, and number of chemotherapy treatments per patient were compared by Mann-Whitney $U$-test.
Univariate analysis was performed by chi-square test, and multivariate analysis was performed by a logistic regression procedure to determine the association between the incidence of ISR and clinical variables. Any variable that was significant by univariate analysis was included as a candidate for multivariate analysis. Data were analyzed with StatView software (SAS Institute, version 5.0, Cary, NC). In this study, $P<0.05$ was considered statistically significant.

\section{RESULTS}

\section{Patients' characteristics}

Overall, 56 patients who underwent FEC, AC or (R-)CHOP regimens were reviewed for this study. There were 7 males and 49 females with an age range of 31-85 years (median age, 50 years). Sixty one injections were administered with fosaprepitant, whereas 98 injections were delivered without fosaprepitant. All injections were administrated through a peripheral vein. There was no statistically significant difference between the 24 patients in the fosaprepitant group and the 32 patients in the control groups with regard to background clinical variables (age, gender, BMI, number of prior chemotherapy regimens and chemotherapy regimens used in the present study) (Table 1).

Table I. Characteristics of Patients

\begin{tabular}{|c|c|c|c|c|}
\hline Variable & $\begin{array}{l}\text { All patients } \\
\text { n (\%) }\end{array}$ & $\begin{array}{l}\text { fAPR(-) } \\
\text { n (\%) }\end{array}$ & $\begin{array}{l}\text { fAPR(+) } \\
\text { n (\%) }\end{array}$ & $P$ value \\
\hline Number of patients & $56(100)$ & $32(100)$ & $24(100)$ & \\
\hline \multicolumn{5}{|l|}{ Age (years) } \\
\hline Median & 50 & 55 & 42 & $0.1494^{*}$ \\
\hline Range & $31-85$ & $32-85$ & $31-69$ & \\
\hline \multicolumn{5}{|l|}{ Gender } \\
\hline Male & $7(13)$ & $3(9)$ & $4(17)$ & $0.4465^{\dagger}$ \\
\hline Female & $49(87)$ & $29(91)$ & $20(83)$ & \\
\hline \multicolumn{5}{|l|}{ Body mass index } \\
\hline Median & 21.3 & 21.1 & 21.3 & $0.9209^{*}$ \\
\hline Range & $15.3-38.8$ & $15.3-38.8$ & $15.6-31.0$ & \\
\hline \multicolumn{5}{|c|}{ Number of prior chemotherapy } \\
\hline 0 & $52(93)$ & $29(91)$ & $23(96)$ & $0.6273^{\dagger}$ \\
\hline 1 & $4(7)$ & $3(9)$ & $1(4)$ & \\
\hline \multicolumn{5}{|c|}{ Chemotherapy regimen } \\
\hline $\mathrm{AC}$ & $24(43)$ & $16(50)$ & $8(33)$ & $0.3499 \ddagger$ \\
\hline FEC & $20(36)$ & $11(34)$ & $9(38)$ & \\
\hline (R-)CHOP & $12(21)$ & $5(16)$ & $7(29)$ & \\
\hline
\end{tabular}

fAPR fosaprepitant; AC, doxorubicin/cyclophosphamide; FEC,

5-fluorouracil/epirubicin/cyclophosphamide;

and (R-)CHOP, rituxi-

mab/cyclophosphamide/doxorubicin/vincristine/prednisolone

$P$ values were determined by * Mann Whitney $U$ test, $\downarrow$ Fisher's exact test, or $\ddagger$ chi-square test. 
Table 2. Characteristics of Injections

\begin{tabular}{|c|c|c|c|c|}
\hline Variable & $\begin{array}{l}\text { All injections } \\
\text { n (\%) }\end{array}$ & $\begin{array}{l}\text { fAPR(-) } \\
\text { n ( } \%)\end{array}$ & $\begin{array}{l}\text { fAPR(+) } \\
\text { n (\%) }\end{array}$ & $\begin{array}{l}P \\
\text { value }\end{array}$ \\
\hline $\begin{array}{l}\text { Number of injec- } \\
\text { tion }\end{array}$ & $159(100)$ & $98(100)$ & $61(100)$ & \\
\hline \multicolumn{5}{|c|}{ Number of injection per patients } \\
\hline Median & 3 & 3 & 3 & \multirow[t]{2}{*}{$0.7795^{*}$} \\
\hline Range & $1-9$ & $1-9$ & $1-8$ & \\
\hline \multicolumn{5}{|c|}{ Chemotherapy regimen } \\
\hline $\mathrm{AC}$ & $64(40)$ & $45(46)$ & $19(31)$ & \multirow[t]{3}{*}{$0.1321^{\dagger}$} \\
\hline FEC & $54(34)$ & $32(33)$ & $22(36)$ & \\
\hline$(\mathrm{R}-) \mathrm{CHOP}$ & $41(26)$ & $21(21)$ & $20(33)$ & \\
\hline \multicolumn{5}{|l|}{ Injection site } \\
\hline Median vein & $3(2)$ & $2(2)$ & $1(2)$ & \multirow[t]{6}{*}{$0.6600^{\dagger}$} \\
\hline Basilic vein & $5(3)$ & $4(4)$ & $1(2)$ & \\
\hline Antebrachial vein & $113(71)$ & $71(73)$ & $42(68)$ & \\
\hline Dorsal hand vein & $2(1)$ & $1(1)$ & $1(2)$ & \\
\hline Radial vein & $34(22)$ & $18(18)$ & $16(26)$ & \\
\hline Unknown & $2(1)$ & $2(2)$ & $0(0)$ & \\
\hline \multicolumn{5}{|c|}{$\begin{array}{l}\text { fAPR fosaprepitant; AC, doxorubicin/cyclophosphamide; FEC, } \\
\text { 5-fluorouracil/epirubicin/cyclophosphamide; }\end{array}$} \\
\hline
\end{tabular}

Baseline characteristics of injections according to the presence or absence of fosaprepitant injection were also compared. The median number of injections per patient was 3 (range 1-8) in the fosaprepitant group and 3 (range 1-9) in the control group (Table 2). There was no significant difference in the number of injections per patient between the two groups. The number of injections by regimen was $19(31 \%)$ with AC, $22(36 \%)$ with FEC, and 20 (33\%) with (R-)CHOP in the fosaprepitant group, and $45(46 \%)$ with AC, 32 (33\%) with FEC, and 21 (21\%) with (R-)CHOP in the control group (Table 2). There was no statistically significant difference in the distribution of chemotherapy regimen and injection sites between the two groups.

\section{The frequency of ISR}

The incidence of ISR per patient and the frequency of ISR per injection with or without fosaprepitant are summarized in Figure 2. The incidence of ISR per patient was $67 \%$ in the fosaprepitant group (16 of 24 patients) and $16 \%$ in the control group (5 of 32 patients). The incidence of patients who experienced ISR was significantly higher in the fosaprepitant group than in the control group $(P=0.0002)$ (Figure 2). The incidence of treatment-required ISR per patient was significantly higher in the fosaprepitant group (12 of 24 patients, $50 \%$ ) compared with the control group (1 of 32 patients, $3.1 \%, P<0.0001$ ).

The frequency of ISR per injection was significantly higher in the fosaprepitant group (21 of 61 injections, 34\%), compared with the control group (8 of
98 injections, $8.2 \%, P<0.0001$ ) (Figure 2). Furthermore, the frequency of treatment-required ISR per injection was significantly higher in the fosaprepitant group (17 of 61 patients, $28 \%$ ) compared with the control group (1 of 98 patients, $1.0 \%, P<0.0001$ ).
(A)

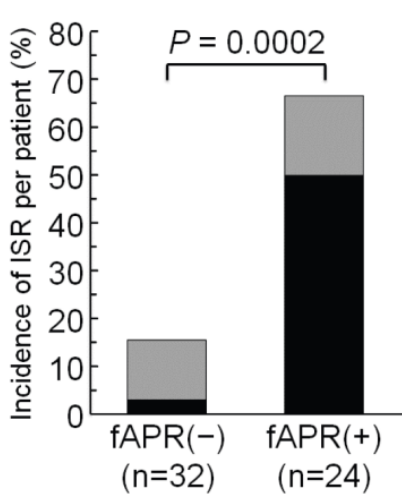

(B)

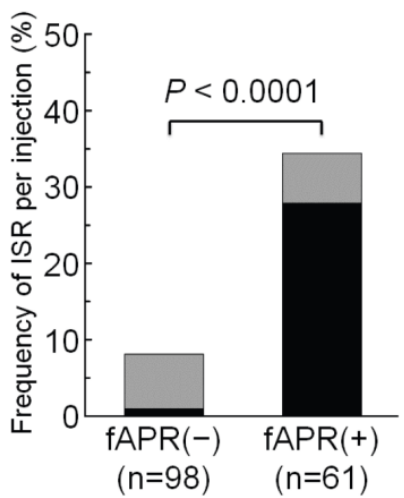

Figure 2. Comparison of the incidence of ISR with or without administration of fosaprepitant: (A) per patient, (B) per injection. ISR was evaluated according to the National Cancer Institute Common Toxicity Criteria (NCl-CTC) version 4.0. Black bars represent the incidence of treatment-required ISR, and gray bars represent the incidence of non-treatment required ISR. ISR, injection site reaction; and fAPR, fosaprepitant.

\section{Clinical symptoms, managements and severities of ISR}

The frequency per injection of clinical symptoms and managements of ISR observed in this study are shown in Figure 3. These items in 61 injections in the fosaprepitant group included pain (14 injections, $23 \%)$, erythema (10 injections, $16 \%)$, swelling (6 injections, $10 \%$ ), delayed drip infusion (the phenomenon observed without the handle of clinical stuffs) (6 injections, $10 \%)$, re-puncture (13 injections, $21 \%$ ), dermatological treatment (12 injections, $20 \%$ ), and discontinuation of the injection (2 injections, $3 \%$ ). In contrast, of 98 injections in the control group, they included pain (6 injections, $6 \%$ ), erythema (1 injection, $1 \%)$, delayed drip infusion (1 injection, $1 \%$ ), and re-puncture (1 injection, $1 \%)$. Dermatological treatment and discontinuation of the injection were not required in any of the cases in the control group.

The incidence of Grade 1 and 2 ISR per patient was $13 \%$ and $54 \%$ in the fosaprepitant group ( 3 and 13 of 24 patients) and $6 \%$ and $9 \%$ in the control group (2 and 3 of 32 patients), respectively (data not shown). The frequency of Grade 1 and 2 ISR per injection was $7 \%$ and $28 \%$ in the fosaprepitant group (4 and 17 of 61 injections) and $2 \%$ and $6 \%$ in the control group ( 2 and 6 of 98 injections), respectively (data not shown). No Grade 3 ISR (ulceration or necrosis; severe tissue damage; operative intervention indicated) was observed in this study. 


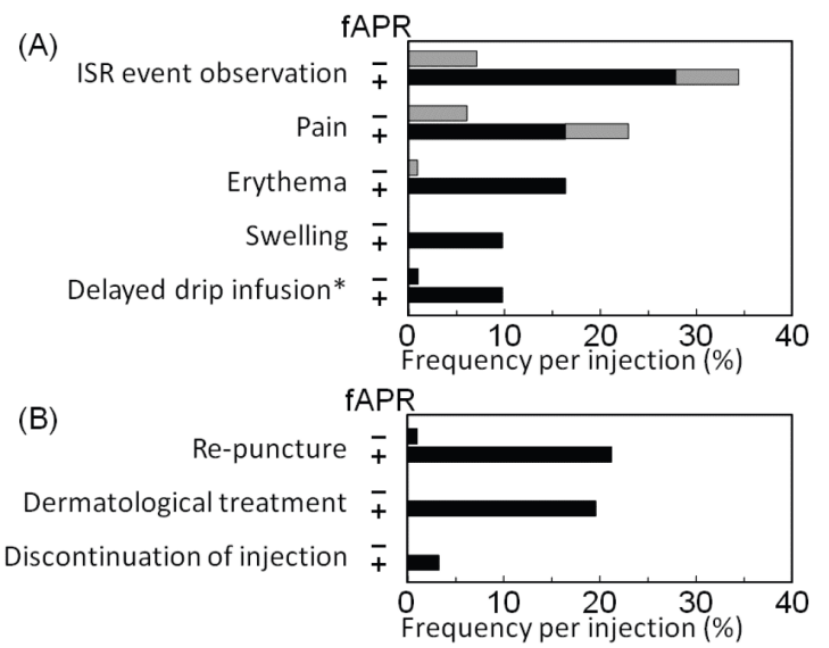

Figure 3. Clinical symptoms (A) and managements of ISR (B). The frequency of (A) clinical symptoms and (B) managements of ISR was assessed for $6 \mathrm{I}$ injections in the fosaprepitant group and 98 injections in the control group. Black bars represent the frequency of symptoms or managements together with treatment-required ISR, and gray bars represent the frequency of clinical symptoms or managements together with non-treatment required ISR. * depicts the phenomenon observed without the handle of clinical staffs. ISR, injection site reaction; and fAPR, fosaprepitant.

\section{Timing of ISR onset in the fosaprepitant group}

In the fosaprepitant group, 20 ISRs were assessable to determine the timing of ISR occurrence. Nine ISRs were observed during the administration of fosaprepitant, and 11 ISRs occurred after the admin- istration of fosaprepitant (data not shown). We also determined the occurrence of ISR with regard to the number of courses of each chemotherapy regimen in 16 fosaprepitant group patients. Four ISRs occurred during the first course, 6 during the second course, 2 during the third course, and 4 during the fourth course (data not shown).

\section{Predictive factors of ISR}

By univariate analysis, an increased risk of ISR per patient was significantly associated with fosaprepitant injection, younger age $(<50$ years), and chemotherapy regimen (Table 3 ). The increased risk of ISR per injection was significantly associated with fosaprepitant administration, younger age $\quad(<50$ years), low BMI $(<22)$, and chemotherapy regimen (Table 3).

By multivariate analysis, fosaprepitant injection and chemotherapy regimen were significant independent variables that correlated with increased risk of ISR per patient (Table 4). Fosaprepitant injection was also a significant independent variable that correlated with increased risk of treatment-required ISR per patient (Table 4). Moreover, fosaprepitant injection was also identified as a significant independent variable that correlated with increased risk of both ISR and treatment-required ISR per injection (Table 5).

Table 3. Relationship between clinical variables and ISRs.

\begin{tabular}{|c|c|c|c|c|c|c|c|c|}
\hline & \multicolumn{4}{|c|}{ ISR incidence per patients } & \multicolumn{4}{|c|}{ ISR frequency per injections } \\
\hline & $\begin{array}{l}\text { All patients } \\
\mathrm{n}\end{array}$ & $\begin{array}{l}\text { ISR(+) } \\
\mathrm{n}(\%)\end{array}$ & $\begin{array}{l}\text { ISR(-) } \\
\mathrm{n}(\%)\end{array}$ & $P$ value & $\begin{array}{l}\text { All injection } \\
\mathrm{n}\end{array}$ & $\begin{array}{l}\text { ISR(+) } \\
\mathrm{n}(\%)\end{array}$ & $\begin{array}{l}\text { ISR(-) } \\
\mathrm{n}(\%)\end{array}$ & $P$ value \\
\hline Number of patients & 56 & $21(38)$ & $35(63)$ & & 159 & $29(18)$ & $130(82)$ & \\
\hline \multicolumn{9}{|l|}{ fAPR injection } \\
\hline Yes & 24 & $16(67)$ & $8(34)$ & $<0.0001$ & 61 & $21(34)$ & $40(66)$ & $<0.0001$ \\
\hline No & 32 & $5(16)$ & $27(84)$ & & 98 & $8(8)$ & $90(92)$ & \\
\hline \multicolumn{9}{|l|}{ Age (years) } \\
\hline$<50$ & 28 & $15(54)$ & $13(46)$ & 0.0130 & 83 & $22(27)$ & $61(73)$ & 0.0048 \\
\hline$\geq 50$ & 28 & $6(21)$ & $22(79)$ & & 76 & $7(9)$ & $69(91)$ & \\
\hline \multicolumn{9}{|l|}{ Gender } \\
\hline Male & 7 & $4(57)$ & $3(43)$ & 0.2511 & 22 & $4(18)$ & $16(82)$ & 0.9940 \\
\hline Female & 49 & $17(35)$ & $32(65)$ & & 137 & $25(18)$ & $112(82)$ & \\
\hline \multicolumn{9}{|l|}{ Body mass index } \\
\hline$<22$ & 35 & $16(46)$ & $19(54)$ & 0.1012 & 102 & $24(24)$ & $78(76)$ & 0.0208 \\
\hline$\geq 22$ & 21 & $5(24)$ & $16(76)$ & & 57 & $5(9)$ & $52(91)$ & \\
\hline \multicolumn{9}{|c|}{ Number of prior chemotherapy } \\
\hline 0 & 52 & $21(40)$ & $31(60)$ & 0.1079 & 150 & $29(19)$ & $121(81)$ & 0.1446 \\
\hline 1 & 4 & $0(0)$ & $4(100)$ & & 9 & $0(0)$ & $9(100)$ & \\
\hline \multicolumn{9}{|c|}{ Chemotherapy regimen } \\
\hline $\mathrm{AC}$ & 24 & $4(17)$ & $20(83)$ & 0.0197 & 64 & $5(8)$ & $59(92)$ & 0.0155 \\
\hline FEC & 20 & $11(55)$ & $9(45)$ & & 54 & $15(28)$ & $39(72)$ & \\
\hline$(\mathrm{R}-) \mathrm{CHOP}$ & 12 & $6(50)$ & $6(50)$ & & 41 & $9(22)$ & $32(78)$ & \\
\hline
\end{tabular}

fAPR, fosaprepitant; ISR, injection site reaction;

AC, doxorubicin/cyclophosphamide; FEC, 5-fluorouracil/epirubicin/cyclophosphamide; and (R-)CHOP, rituxi-

$\mathrm{mab} /$ cyclophosphamide/doxorubicin/vincristine/prednisolone

$P$ values were determined by chi-square test. 
Table 4. Multivariate Logistic Regression Analysis for ISR incidence per patients.

\begin{tabular}{lcc}
\hline Variable & ISR incidence per patients \\
\cline { 3 - 3 } & Odd Ratio (95\%CI) & $P$ value \\
\hline Incidence of ISR & $10.476(2.282-48.091)$ & 0.0025 \\
Fosaprepitant injection (Yes vs. No) & $2.177(0.424-11.183)$ & 0.3514 \\
Age (<50 vs. $\geq 50)$ & $4.833(0.725-32.202)$ & 0.1034 \\
Chemotherapy regimen (AC vs (R-)CHOP) & $6.183(1.019-37.507)$ & 0.0476 \\
Chemotherapy regimen (AC vs FEC) & & 0.0031 \\
Incidence of treatment-required ISR & $31.979(3.220-317.621)$ & 0.7567 \\
Fosaprepitant injection (Yes vs. No) & $0.731(0.101-5.293)$ & 0.1877 \\
Age (<50 vs. $\geq 50)$ & $3.805(0.521-27.793)$ & 0.4130 \\
Chemotherapy regimen (AC vs (R-)CHOP) & $2.378(0.299-18.913)$ & \\
Chemotherapy regimen (AC vs FEC) &
\end{tabular}

CI, confidence intervals; ISR, injection site reaction; AC, doxorubicin/cyclophosphamide; (R-)CHOP, rituximab/cyclophosphamide/doxorubicin/vincristine/prednisolone; and FEC, 5-fluorouracil/epirubicin/cyclophosphamide.

Table 5. Multivariate Logistic Regression Analysis for ISR frequency per injections.

\begin{tabular}{llc}
\hline Variable & ISR frequency per patients \\
\cline { 2 - 3 } & Odd Ratio (95\%CI) & $P$ value \\
\hline Frequency of ISR & $4.565(1.750-11.908)$ & 0.0019 \\
Fosaprepitant injection (Yes vs. No) & $1.615(0.525-4.970)$ & 0.4031 \\
Age (<50 vs. $\geq 50)$ & $2.810(0.799-9.882)$ & 0.1073 \\
Chemotherapy regimen (AC vs (R-)CHOP) & $3.401(0.951-12.169)$ & 0.0598 \\
Chemotherapy regimen (AC vs FEC) & $2.235(0.729-6.854)$ & 0.1594 \\
Body mass index (<22 vs. $\geq 22)$ & & 0.0010 \\
Frequency of treatment-required ISR & $33.197(4.113-267.916)$ & 0.9234 \\
Fosaprepitant injection (Yes vs. No) & $0.934(0.231-3.776)$ & 0.1660 \\
Age $(<50$ vs. $\geq 50)$ & $2.882(0.645-12.884)$ & 0.6065 \\
Chemotherapy regimen (AC vs (R-)CHOP) & $1.556(0.289-8.381)$ & 0.6289 \\
Chemotherapy regimen (AC vs FEC) & $1.410(0.350-5.687)$ & \\
Body mass index $(<22$ vs. $\geq 22)$ & & \\
\hline
\end{tabular}

CI, confidence intervals; ISR, injection site reaction; AC, doxorubicin/cyclophosphamide;

(R-)CHOP, rituximab/cyclophosphamide/doxorubicin/vincristine/prednisolone;

and FEC, 5-fluorouracil/epirubicin/cyclophosphamide.

Control group

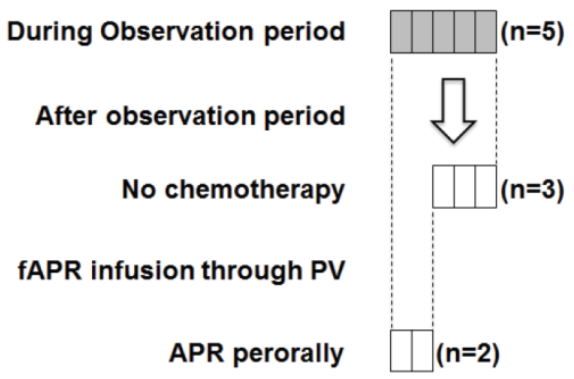

fAPR infusion through CV
Fosaprepitant group

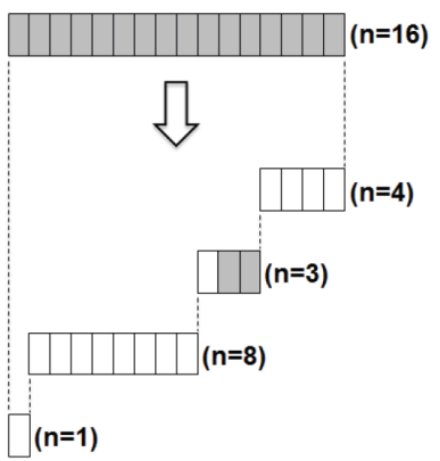

no ISR ISR

Figure 4. The ISR monitoring of patients who experienced ISR during or after the observation period. After the observation period, we monitored the occurrence of ISR in patients who experienced ISR during the observation period. Patients who experienced ISR from fosaprepitant injection did not develop ISR again when they received subsequent chemotherapy with peroral administration of aprepitant. Gray panels indicate patients who experienced ISR; and white, patient had no ISRs. fAPR, fosaprepitant; APR, aprepitant; PV, peripheral vein; CV, central venous; and ISR, injection site reaction.

\section{Occurrence of ISR after the investigation period}

After the investigation period (November 2011 to April 2012), we monitored patient records for the occurrence of ISR in those who had experienced ISR during the investigation period (Figure 4). In the control group, ISRs occurred in 5 patients during the ob- 
servation period, and none of the ISRs resulted in discontinuation of chemotherapy. After the investigation period, 3 of 5 patients did not require further administration because their prescribed treatments were completed. The other 2 patients who continued chemotherapy with aprepitant orally experienced no ISRs even when chemotherapy was continued.

In the fosaprepitant group, ISRs occurred in 16 patients during the investigation period, and none of the ISRs resulted in discontinuation of chemotherapy. After the investigation period, 4 of 16 patients did not require further administration because their prescribed treatments were completed, and another 12 patients continued chemotherapy. In two patients, ISRs reoccurred when they were administered chemotherapy with fosaprepitant through a peripheral vein, while one patient had no ISRs even when chemotherapy with fosaprepitant was continued. When the administration route was changed from peripheral injection (fosaprepitant) to oral (aprepitant) in 8 patients, they did not suffer ISRs. One patient whose administration route was changed from peripheral injection to central venous injection did not develop ISRs.

\section{DISCUSSION}

The present study suggested that ISRs were induced at a high frequency and were enhanced when fosaprepitant was injected through a peripheral vein in patients treated with an anthracycline-containing regimen.

In the present study, the incidence of ISR was significantly higher in the fosaprepitant group patients $(16 / 24,66.7 \%)$ compared with the control group patients $(5 / 32,16 \%)$, and was consistent with previous reports. $5,8,9$ The incidence of fosaprepitant-induced ISR was also higher in the present study than in previous studies, $, 8,8$ although the incidence of ISR could not be compared directly due to a difference in the number of fosaprepitant administrations. The percentage of ISR was $2.2 \%$ in patients who received a single dose of fosaprepitant and cisplatin enrolled into the Ease-017 (Evaluation of fosAprepitant in Single-dose schedulE) trial, $5.9 \%$ in an interview form released by the pharmaceutical company that supplies fosaprepitant in Japan, ${ }^{8}$ and $23.6 \%$ in a Japanese phase 3 trial of patients who received a single dose of fosaprepitant and cisplatin. ${ }^{9}$ Additionally, 15 of 32 $(46.9 \%)$ young healthy volunteers experienced ISR when they were injected with $200 \mathrm{mg}(1 \mathrm{mg} / \mathrm{mL})$ of fosaprepitant alone. ${ }^{10}$ The percentage of epirubicin-induced local skin toxicity was $19.5 \% 11$, and this was similar to the ISR incidence of patients who were not administered fosaprepitant in the present study. The major difference between previous reports and our study was that all patients enrolled in our study were administered anthracycline-containing chemotherapy, whereas most patients enrolled in previous studies received cisplatin based-chemotherapy. This indicated that anthracyclines coadministered with fosaprepitant have the potential to cause greater tissue damage, rather than coadministration with cisplatin, especially as anthracyclines commonly cause severe extravasation. ${ }^{12}$ Moreover the incidence of treatment-required ISR, defined as ISR that required re-puncture, dermatological treatment (consultation with a dermatologist, the application of steroid ointment, or local injection to the ISR site), and/or discontinuation of administration in present study, was significantly higher in the fosaprepitant group. Thus, the strong venous toxicity of anthracyclines can cause and worsen fosaprepitant-induced ISR and increase ISR frequency and severity.

Another difference between previous reports and our study is the concentration of fosaprepitant used. The incidence and severity of injection site pain caused by fosaprepitant was associated with the concentration and dosing rate of fosaprepitant. ${ }^{8}$ In the present study, fosaprepitant was administered using the highest concentration $(1.5 \mathrm{mg} / \mathrm{mL})$ and fastest speed ( $30 \mathrm{~min}$ ) recommended by the manufacturers in Japan, and this might have raised the risk of ISR development. In comparison, manufacturers in the US recommend fosaprepitant is dissolved in $150 \mathrm{~mL}(1.0$ $\mathrm{mg} / \mathrm{mL}$ ) of normal saline and infused over 20-30 min. Thus, the fosaprepitant concentration used in Japan is about 1.5 times higher than in the US. Therefore, one reason why ISRs are common in Japan might be the use of higher concentrations of fosaprepitant compared with other countries. Imazu et al. reported that the frequency of ISR was decreased to $13.3 \%$ from $52.9 \%$ after reducing the concentration of fosaprepitant from $1.5 \mathrm{mg} / \mathrm{mL}$ to $0.6 \mathrm{mg} / \mathrm{mL}$ in Japanese cancer patients. $^{13}$ In addition, hydration in cisplatin-containing regimens could reduce the concentration of fosaprepitant, and might diminish the frequency of fosaprepitant-induced ISR.

Standard prophylaxis to reduce the incidence and severity of ISR during/after fosaprepitant administration has not yet been established. In the present study, patients who experienced ISR from fosaprepitant injection did not develop ISR again when they received subsequent chemotherapy with peroral administration of aprepitant. Thus, if peroral administration of drugs is possible, we would recommend oral administration of aprepitant instead of fosaprepitant injection through a peripheral vein during chemotherapy including anthracycline. In contrast, in cases where patients cannot intake the drug orally, it is preferable to use fosaprepitant. The higher the 
concentration of fosaprepitant, the higher the incidence of ISR. 8,13 Thus, if a more dilute concentration of fosaprepitant is injected slowly, the incidence and severity of ISR could be reduced, although further study is required to confirm this hypothesis.

There are several limitations in the present study. First, this study was a retrospective study using a small diverse clinical population where the data were collected in a clinical context. However, the patient groups in this study were quite homogenous as all patients had a punctured vessel just before fosaprepitant administration, and were infused with the same concentration and rate of fosaprepitant. The dosing procedure of anticancer drugs used in particular chemotherapy regimens was also the same in the two study facilities. We focused on patients at ambulatory treatment centers where well-trained oncology nurses were resident, since both inpatients and clinical ward staff were quite heterogeneous. However, selection bias could not be avoided. To reduce bias, we analyzed all patients who were administrated fosaprepitant through a peripheral vein during the study period. Moreover, there were some deviations in distribution such as cancer type and gender, because anthracycline anticancer agents are commonly used for breast cancer treatment. A second limitation of our study was that the influence of anthracycline on fosaprepitant-associated ISR was too low to evaluate, since there were two outpatients treated with the non-anthracycline regimen together with fosaprepitant at our ambulatory treatment centers during study period. Furthermore, it is unknown whether fosaprepitant-induced ISR occurred prior to starting anthracycline (or vincristine) in 11 ISRs that occurred after drip administration of fosaprepitant, because the correct emergence time of ISR was not clear in the patients' medical records. Although anthracycline and other anti-cancer drugs such as vincristine and cyclophosphamide might cause ISR, the present study indicated that the incidence and severity of ISR was worse in the fosaprepitant group. Therefore, fosaprepitant might affect subsequent injections. To evaluate further the ISR risk caused by fosaprepitant and anthracycline, a prospective randomized controlled study is required. However, this might not be advisable because ISR can be avoided by oral administration of aprepitant instead of fosaprepitant injection. In addition, it is unethical to provide placebo instead of anthracycline because anthracyclines are key drugs for the treatment of breast cancer and hematological malignancies. However, this study identified high ISR risk factors and proposed a workaround in anthracycline-treated patients together with peripheral venous injection of fosaprepitant, although the present study was a small retrospective study.
In conclusion, increased frequency and severity of ISR were caused when fosaprepitant was injected through a peripheral vein in patients treated with anthracyclines. Accordingly, peripheral vein injection of fosaprepitant should be avoided in patients receiving anthracycline-containing chemotherapy. Further studies are required to clarify the underlying mechanisms of ISR and to establish a standard prophylaxis of ISR associated with fosaprepitant administration.

\section{Acknowledgments}

The authors thank the following colleagues for the clinical care of patients: Etsuko Aoki, Yumiko Chigusa, Michiyo Goto, Emi Tanaka, Miki Osaki, Asami Masuda and Yuka Kishi. The authors also thank the following colleagues for their kind advice on the study design, statistical analyses, and evaluation of the results: Masato Nakao, Satoshi Morita, Noriyo Kaneko and Seichi Ichikawa.

Funding sources: This work was supported by Training expenses of Nagoya City West Medical Center.

\section{Competing Interests}

The authors have declared that no competing interest exists.

\section{References}

1. Hesketh PJ, Grunberg SM, Gralla RJ, et al. The oral neurokinin-1 antagonist aprepitant for the prevention of chemotherapy-induced nausea and vomiting: a multinational, randomized, double-blind, placebo-controlled trial in patients receiving high-dose cisplatin - the Aprepitant Protocol 052 Study Group. J Clin Oncol. 2003; 21: 4112-9.

2. Poli-Bigelli S, Rodrigues-Pereira J, Carides AD, et al. Addition of the neurokinin-1 receptor antagonist aprepitant to standard antiemetic therapy improves control of chemotherapy induced nausea and vomiting: results from a randomized, double-blind, placebo-controlled trial in Latin America. Cancer. 2003; 97: 3090-8.

3. Warr DG, Hesketh PJ, Gralla RJ, et al. Efficacy and tolerability of aprepitant for the prevention of chemotherapy-induced nausea and vomiting in patients with breast cancer after moderately emetogenic chemotherapy. J Clin Oncol. 2005; 23: 2822-30.

4. Lasseter KC, Gambale J, Jin B, et al. Tolerability of fosaprepitant and bio equivalency to aprepitant in healthy subjects. J Clin Pharmacol. 2007; 47: 834-40.

5. Grunberg S, Chua D, Maru A, et al. Single-Dose fosaprepitant for the prevention of chemotherapy-induced nausea and vomiting associated with cisplatin therapy: randomized, double-blind study protocol-EASE. J Clin Oncol. 2011; 29: 1495-501.

6. Basch E, Prestrud AA, Hesketh PJ, et al. Antiemetics: American society of clinical oncology guideline update. J Clin Oncol. 2011; 29: 4189-98.

7. Roila F, Herrstedt J, Aapro M, et al. Guideline update for MASCC and ESMO in the prevention of chemotherapy- and radiotherapy-induced nausea and vomiting: results of the Perugia consensus conference. Ann Oncol. 2010; 21 (Suppl. 5): v232-v243.

8. [Internet] Ono Pharmaceutical Co., Ltd. Proemend for intravenous infusion $150 \mathrm{mg}$, Interview Form [In Japanese]. http://www.info.pmda.go.jp/go/interview/ 1/180188_2391405D1020_1_005_1F.pdf

9. Saito H, Yoshizawa H, Yoshimori K, et al. Efficacy and safety of single-dose fosaprepitant in the prevention of chemotherapy-induced nausea and vomiting in patients receiving high-dose cisplatin: a multicentre, randomised, double-blind, placebo-controlled phase 3 trial. Ann Oncol. 2013; 24: 1067-73.

10. Marbury TC, Jin B, Murphy MG, et al. Lack of effect of aprepitant or its prodrug fosaprepitant on QTc intervals in healthy subjects. Anesth Analg. 2009; 109: 418-25.

11. [Internet] Sagent Pharmaceuticals, Schaumburg IL. Epirubicin Hydrochloride Injection [Package insert]; 2011. http://www.sagentpharma.com/Products/ Epirubicin/Catalog/Epirubicin_PI.pdf

12. Langstein HN, Duman H, Seelig D, Butler CE, Evans GR. Retrospective study of the management of chemotherapeutic extravasation injury. Ann Plast Surg. 2002; 49: 369-74.

13. Imazu K, Shinozaki K, Kinoshita M, et al. Investigation of the incidence of Fosaprepitant-induced vascular pain toward prevention. J Jpn Soc Hosp Pharm. 2013; 49: 635-9. 